Check for updates

Cite this: RSC Adv., 2018, 8, 11834

Received 26th February 2018

Accepted 20th March 2018

DOI: $10.1039 / \mathrm{c} 8 \mathrm{ra01709c}$

rsc.li/rsc-advances

\title{
Phosphonium acidic ionic liquid: an efficient and recyclable homogeneous catalyst for the synthesis of 2-arylbenzoxazoles, 2-arylbenzimidazoles, and 2-arylbenzothiazoles $\dagger$
}

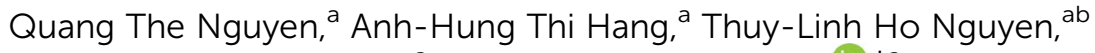 \\ Duy-Khiem Nguyen $\mathrm{Chau}^{\mathrm{a}}$ and Phuong Hoang Tran (D) *a
}

\begin{abstract}
A highly efficient and green strategy for the synthesis of 2-arylbenzoxazoles, 2-arylbenzimidazoles, and 2arylbenzothiazoles catalyzed by phosphonium acidic ionic liquid has been developed via the condensation of o-aminophenol, o-phenylenediamines, and o-aminothiophenol, respectively, with aldehydes. The reaction has a good yield, the broad substrate scope, and mild condition. Triphenyl(butyl-3-sulphonyl) phosphonium toluenesulfonate catalyst was easily obtained from cheap and available starting materials through a one-pot synthesis. Its structure was identified by ${ }^{1} \mathrm{H} N M R,{ }^{13} \mathrm{C} \mathrm{NMR},{ }^{31} \mathrm{P} N M R$, and FT-IR techniques. Other properties including thermal stability and acidity were determined by TGA and Hammett acidity function method. Interestingly, the catalyst can maintain its constantly outstanding performance till the fourth recovery.
\end{abstract}

\section{Introduction}

Benzoxazole, benzimidazole, and benzothiazole are well-known heterocyclic scaffolds commonly found in various anticancer, antimicrobial, antidiabetic, anti-inflammatory, antioxidant, anticonvulsant, and analgesic agents due to their widespectrum biological and pharmaceutical properties. ${ }^{\mathbf{1 - 1 2}}$ To date, there have been two pathways for the preparation of these compounds. ${ }^{13}$ The first one relying on the metal-catalyzed crosscoupling is more competent. ${ }^{\mathbf{1 4}}$ However, it has several drawbacks such as expensive catalysts, ${ }^{15}$ addition of auxiliary reagents, ${ }^{\mathbf{1 6}}$ harsh reaction condition, ${ }^{\mathbf{1 7}}$ prolonged reaction time, ${ }^{18}$ low yields, ${ }^{18}$ volatile organic solvents, ${ }^{19}$ which cause a greatly negative impact on the environment as well as an impediment in the scaling up for industrial production. The second synthetic pathway involves the condensation of 2-aminophenol with aldehydes or carboxylic acids under acidic condition. ${ }^{7,20-24}$ Similarly to metal-catalyzed cross-couplings, major defects including the use of expensive chemicals and volatile organic solvents, long reaction times, and tedious workup procedures have been still unsolvable. Therefore, the development of an efficient, reusable, and green catalyst is

${ }^{a}$ Department of Organic Chemistry, Faculty of Chemistry, University of Science, Vietnam National University - Ho Chi Minh City, 721337, Viet Nam. E-mail: thphuong@hcmus.edu.vn

${ }^{b}$ Center for Innovative Materials and Architectures, Vietnam National University - Ho Chi Minh City, 721337, Viet Nam

† Electronic supplementary information (ESI) available. See DOI: 10.1039/c8ra01709c eventually favorable to facilitate the large-scale production of these important heterocyclic compounds.

In recent years, ionic liquids (ILs) have received increasing interest as environmentally benign media owing to their special properties such as thermal stability, biodegradability, and nonvolatility. ${ }^{25-29}$ They have been widely used as catalysts for a large number of organic transformations..$^{30-39}$ However, their application as catalysts for the synthesis of 2-arylbenzoxazole, 2arylbenzimidazoles, and 2-arylbenzothiazoles via the condensation of aldehydes with $o$-aminophenols, $o$-phenylenediamine, and $o$-aminothiophenols, respectively, has not been known in the literature. In the continuation of our study in ionic liquids application, we reported herein the use of phosphonium acidic ionic liquid as a green and efficient catalyst for the synthesis of the above mentioned arylated heterocycles. The merits of this method are short reaction time, high yield, wide substrate scope, and recyclability of the catalyst.

\section{Results and discussion}

Initially, the condensation of $o$-aminophenol and benzaldehyde is chosen as the model reaction for optimization study. The results were shown in Table 1.

The effect of temperature, reaction time, and phosphonium ionic liquid loading was investigated by conducting the reaction at various values of each parameter (r.t. $-120{ }^{\circ} \mathrm{C}, 20-360 \mathrm{~min}$, and $0-10 \mathrm{~mol} \%$ ). It was indicated that best condition at $100{ }^{\circ} \mathrm{C}$, $20 \mathrm{~min}$, and $7 \mathrm{~mol} \%$ of catalyst could afford the desired 2 phenylbenzoxazole in an excellent yield of $91 \%$. In the absence 


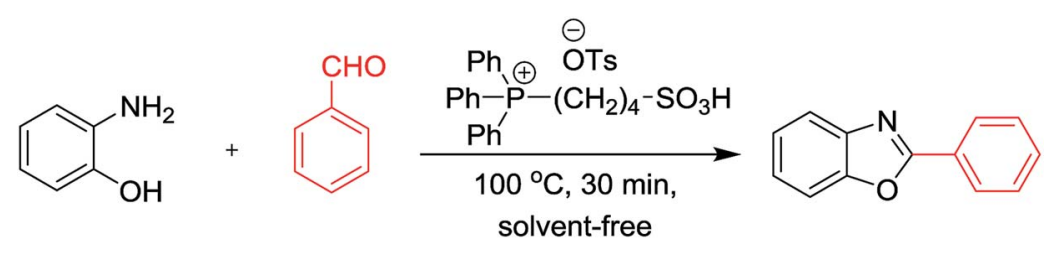

\begin{tabular}{lllll}
\hline Entry $^{a}$ & Catalyst, $(\mathrm{mol} \%)$ & Time, $(\mathrm{min})$ & ${\text { Temperature, }\left({ }^{\circ} \mathrm{C}\right)}$ \\
\hline 1 & 10 & 360 & r.t. & Trace \\
2 & 10 & 360 & 60 & 47 \\
3 & 10 & 90 & 80 & 85 \\
4 & 10 & 20 & 100 & 75 \\
5 & 10 & 30 & 100 & 92 \\
6 & 10 & 40 & 100 & 94 \\
7 & 10 & 20 & 120 & 89 \\
8 & 7 & 30 & 100 & 91 \\
9 & 6 & 30 & 100 & 82 \\
10 & 5 & 30 & 100 & 75 \\
11 & 0 & 360 & 100 & -
\end{tabular}

${ }^{a}$ Reaction conditions: 2-aminophenol $(1 \mathrm{mmol})$, benzaldehyde $(1 \mathrm{mmol})$, solvent-free. ${ }^{b}$ Isolated yields by column chromatography (acetone/ petroleum ether $=1 / 19$ ).

of the catalyst, the failure in the formation of this product was observed as a result.

Next, different catalysts were tested for their performance in the synthesis of 2-phenylbenzoxazole (see detailed experiments in the ESI $\dagger$ ). The desired product was achieved in the best yield using phosphonium acidic ionic liquid while much lower yields were observed in the same reaction mediated by other catalysts.

With the optimal condition in hand, we explored the reaction scope over a large number of aldehydes in the formation of 2-arylbenzoxazoles and other analogues (Table 2). In general, the reactions proceeded smoothly to give the corresponding products in good to excellent yields. Intuitively, the electronic properties of substituents of benzaldehydes exhibited a little effect on the reaction. As a common trend, electron-rich aldehydes gave the expected products in slightly better yields than electron-poor analogues. Only substrates bearing a powerful electron-withdrawing group such as $p$-nitro or $o$-fluoro can give rise to a dramatic yield decrease as low as $68-78 \%$ (entry 7,10 , 41, 45). Similarly, the substituents on 2-aminophenol have certain effect on the yields of the desired products. For 2-aminophenol bearing an electron-donating or a weak electronwithdrawing group such as methyl or chloro, respectively, their condensation with a variety of aldehydes could easily approach the completion with the yields of arylated products above $80 \%$. However, a sharply adverse impact was seen for 2 amino-4-nitrophenol whose arylated products can be only isolated in lower yields of $74-81 \%$ compared to $81-93 \%$ reported for non-substituted 2-aminophenol (entries 30-35 and 1-6). Meanwhile, on examining the reactivity for a row consisting of eight common aldehydes (entries 14-21 and 22-29), both 2amino-4-methylphenol and 2-amino-4-chlorophenol can be converted to corresponding heterocyclic adducts in closely similar yields (85-92\% for 2-aryl-5-methylbenzoxazoles and 86-
$96 \%$ for 2-aryl-5-chlorobenzoxazoles) compared to those reported in the arylation of non-substituted 2-aminophenol (81$93 \%$ ). For some arylations in which 2-amino-4-nitrophenol or 4nitrobenzaldehyde was employed as starting materials, the unexpected solidification of the ongoing reaction mixture can slow down the reaction rate as the result of heterogeneity. Therefore, adding a small amount of DI water $(0.2 \mathrm{~mL})$ to the reaction mixture in combination with increasing temperature to $120{ }^{\circ} \mathrm{C}$ can be helpful to significantly facilitate the reaction (entries 30, 41, 45).

As our expectation, this synthetic campaign is well-suited for the preparation of not only 2-arylbenzoxazoles but also other heterocyclic analogues including 2-arylbenzimidazoles and 2arylbenzothiazoles. While 2-aminothiophenol was able to undergo the arylation with the same ease as 2-aminophenol, $o$ phenylenediamine required a prolonged reaction time up to $90 \mathrm{~min}$ for a quantitative transformation into expected 2-arylbenzimidazoles. Nevertheless, this harsh condition did not work out in every case. For instance, the arylation of $o$-phenylenediamine by less reactive 4-nitrobenzaldehyde only afforded the desired product in 68\% yield even as a small amount of water was added to the reaction mixture to maintain the homogeneity.

Phosphonium acidic ionic liquid was readily prepared via one-pot two-step procedure in high yield and used as the catalyst for esterification. ${ }^{40}$ However, to the best of our knowledge, no application of this ionic liquid as the catalyst for the condensation between 2-aminophenol, $o$-phenylenediamines or 2-aminothiophenol with aldehydes has been reported in the literature so far. Fig. 1 shows the thermal gravimetric analysis (TGA) of triphenyl(butyl-3-sulphonyl)phosphonium toluenesulfonate. The first weight loss of $11.64 \%$ below $200{ }^{\circ} \mathrm{C}$ merely corresponds to the loss of residual water in the sample. The 
Table 2 Synthesis of 2-arylbenzoxazoles, 2-arylbenzimidazoles, and 2-arylbenzothiazoles catalyzed by $\left[\left(\mathrm{C}_{6} \mathrm{H}_{5}\right)_{3} \mathrm{P}\left(\mathrm{CH}_{2}\right)_{4} \mathrm{SO}{ }_{3} \mathrm{H}\right][\mathrm{OTs}]^{a}$

\begin{tabular}{|c|c|c|c|c|c|}
\hline 1 & 1a: $\mathbf{R}=\mathbf{H}, \mathbf{X}=\mathbf{O H}, 2 \mathrm{a}: \mathrm{Ar}=\mathrm{Ph}$ & & 100 & 30 & 91 \\
\hline 2 & 2b: $\mathrm{Ar}=4-\mathrm{MeC}_{6} \mathrm{H}_{4}$ & & 100 & 45 & 90 \\
\hline 4 & $2 \mathrm{~d}: \mathrm{Ar}=4-\mathrm{MeOC}_{6} \mathrm{H}_{4}$ & & 100 & 50 & 93 \\
\hline 5 & 2e: $\mathrm{Ar}=4-\mathrm{FC}_{6} \mathrm{H}_{4}$ & & 100 & 45 & 81 \\
\hline 6 & $2 \mathrm{f}: \mathrm{Ar}=4-\mathrm{ClC}_{6} \mathrm{H}_{4}$ & & 100 & 45 & 91 \\
\hline $7^{c}$ & $2 \mathrm{~g}: \mathrm{Ar}=4-\mathrm{O}_{2} \mathrm{NC}_{6} \mathrm{H}_{4}$ & & 120 & 50 & 75 \\
\hline 10 & $2 \mathrm{j}: \mathrm{Ar}=2-\mathrm{FC}_{6} \mathrm{H}_{4}$ & & 100 & 45 & 78 \\
\hline 11 & $2 \mathrm{k}: \mathrm{Ar}=2-\mathrm{ClC}_{6} \mathrm{H}_{4}$ & & 100 & 45 & 90 \\
\hline 12 & 21: $\mathrm{Ar}=2-\mathrm{HOC}_{6} \mathrm{H}_{4}$ & & 120 & 50 & 90 \\
\hline 13 & $2 \mathrm{~m}: \mathrm{Ar}=4$-pyridinyl & & 100 & 50 & 82 \\
\hline 14 & 1b: $\mathbf{R}=\mathbf{M e}, \mathbf{X}=\mathbf{O H}, 2 \mathrm{a}: \mathrm{Ar}=\mathrm{Ph}$ & & 100 & 35 & 90 \\
\hline
\end{tabular}




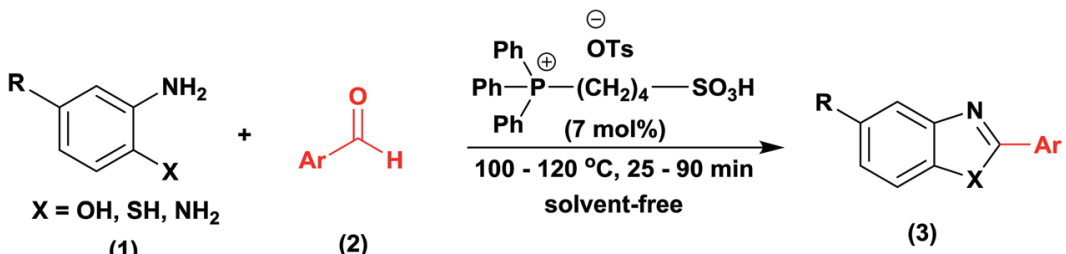

(1)

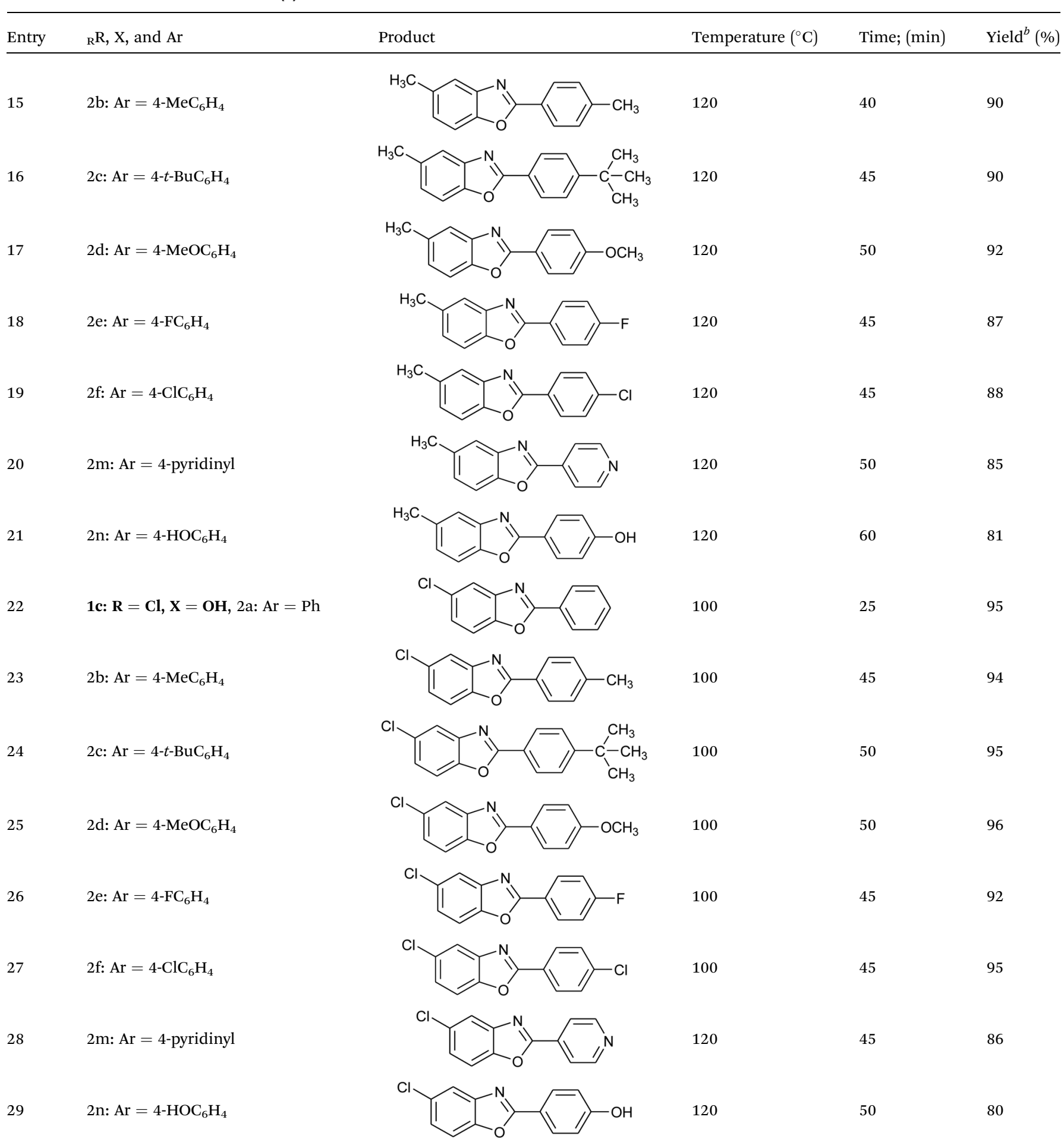




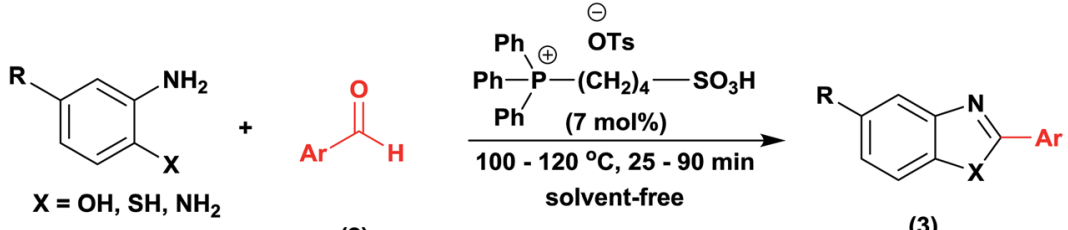

(1)

(2)

(3)

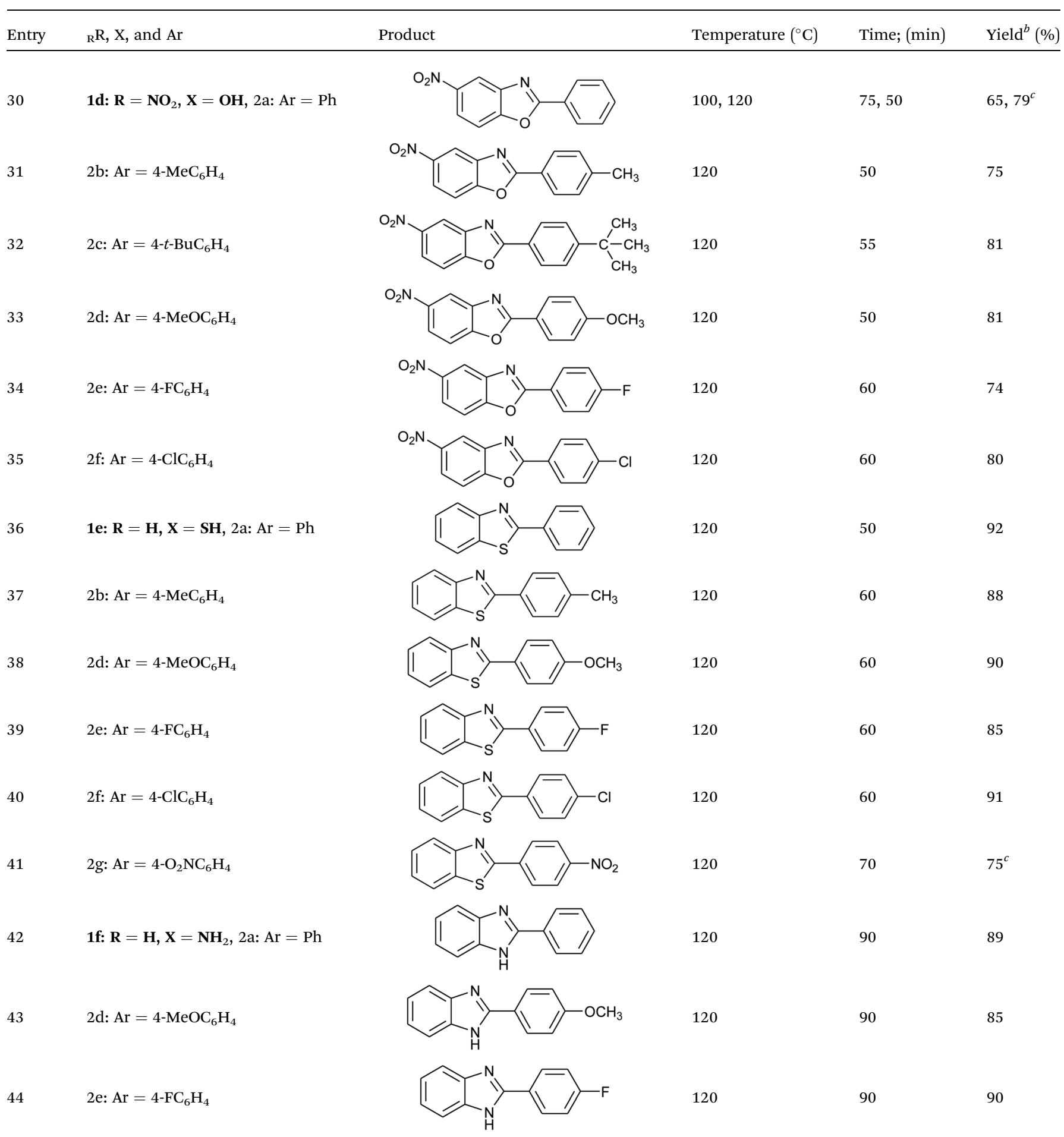


Table 2 (Contd.)

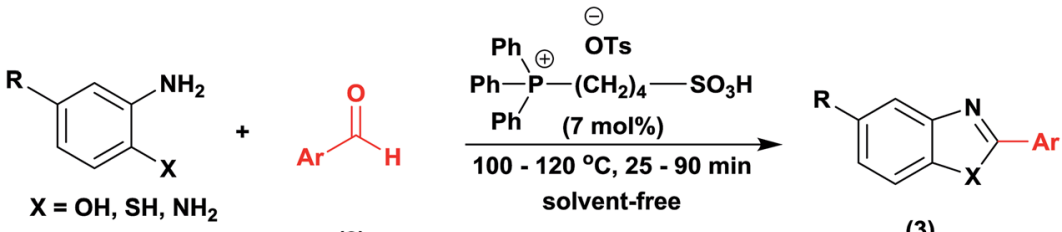

(1)

(2)

(3)

\begin{tabular}{lllll}
\hline Entry & ${ }_{\mathrm{R}} \mathrm{R}, \mathrm{X}$, and $\mathrm{Ar}$ & Temperature $\left({ }^{\circ} \mathrm{C}\right)$ & Time; (min) & Yield ${ }^{b}(\%)$ \\
\hline 5 & $2 \mathrm{~g}: \mathrm{Ar}=4-\mathrm{O}_{2} \mathrm{NC}_{6} \mathrm{H}_{4}$ & & & \\
\hline
\end{tabular}

\begin{abstract}
${ }^{a}$ Reaction conditions: 2-aminophenol (1 mmol), or 2-aminothiophenol (1 mmol), or $o$-phenylenediamine $(1 \mathrm{mmol})$; aldehyde $(1 \mathrm{mmol})$; solventfree. ${ }^{b}$ Isolated yields by column chromatography (acetone/petroleum ether $=1 / 19$ or ethyl acetate/hexanes $\left.=1 / 19\right) .{ }^{c}$ Water $(0.2 \mathrm{~mL})$ was added to the reaction mixture.
\end{abstract}

phosphonium acidic ionic liquid then maintains its stability up to $220-225{ }^{\circ} \mathrm{C}$ before undergoing two consecutive thermal decomposition steps at $225-400{ }^{\circ} \mathrm{C}$ and $400-600{ }^{\circ} \mathrm{C}$ corresponding to the weight loss of 76.75 and $8.81 \%$, respectively. From the data of TGA as well as latter FT-IR analysis (Fig. 2), it could be firmly assured that there is no structural deformation of the ionic liquid at the temperature range of the investigated arylation of benzoxazole and other analogues $\left(100-120^{\circ} \mathrm{C}\right)$.

The FT-IR spectra of primitively prepared triphenyl(butyl-3sulphonyl)phosphonium toluenesulfonate and its recovered sample are presented in Fig. 2 . The broad peak at approximately $3450 \mathrm{~cm}^{-1}$ indicates the presence of hydroxyl group while overlapped shoulder peaks scattered from 2950 to $3100 \mathrm{~cm}^{-1}$ are assigned to alkyl and aromatic $\mathrm{C}-\mathrm{H}$ stretching vibrations. The absorption band at about $1600-1486 \mathrm{~cm}^{-1}$ is paired with

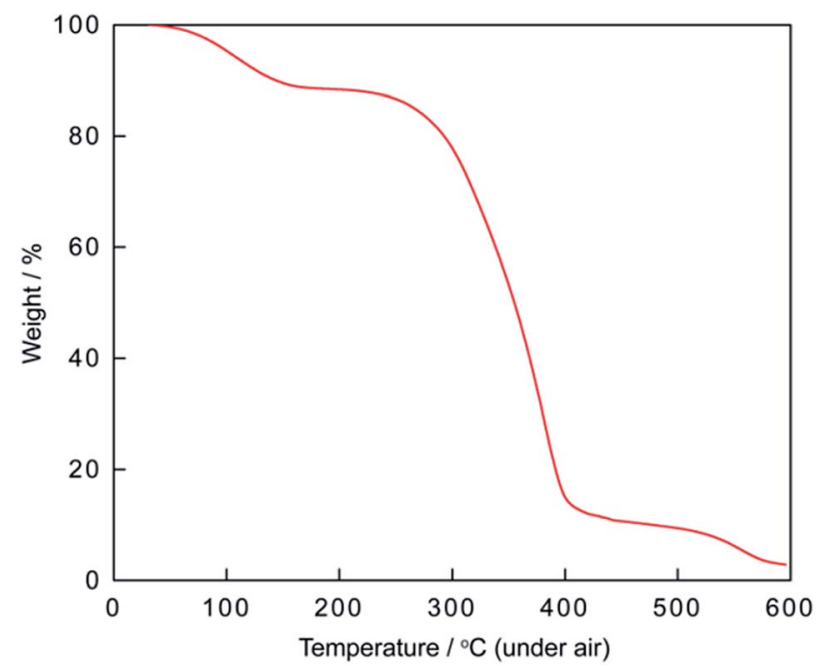

Fig. 1 TGA curve of triphenyl(butyl-3-sulphonyl)phosphonium toluenesulfonate. a number of $\mathrm{C}=\mathrm{C}$ stretching vibrations in aromatic rings. The signals at $1200 \mathrm{~cm}^{-1}$ and $1034 \mathrm{~cm}^{-1}$ are asymmetric and symmetric stretching vibrations of $\mathrm{S}=\mathrm{O}$ bond, respectively.

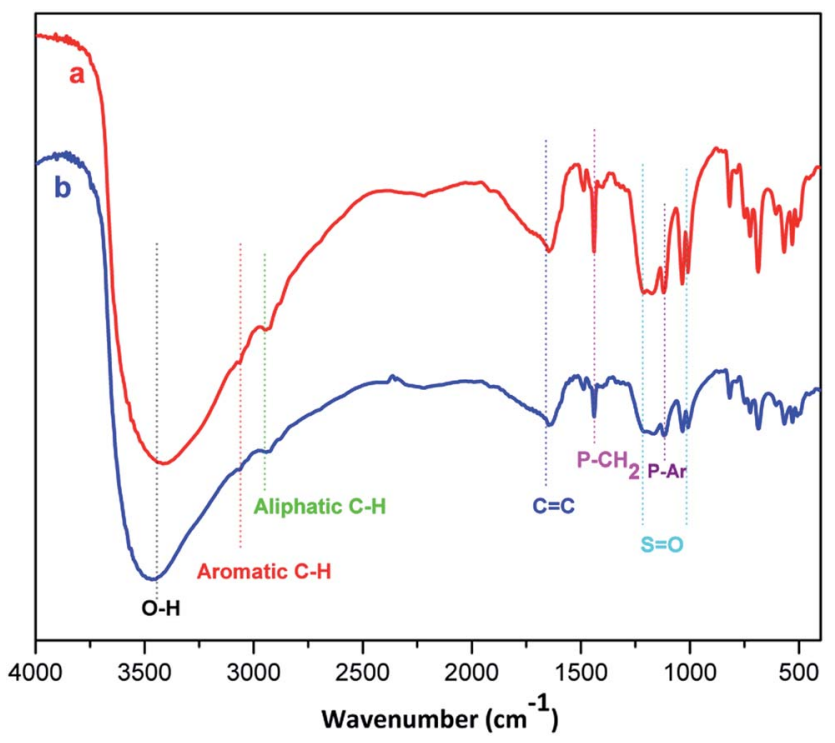

Fig. 2 FT-IR spectra of triphenyl(butyl-3-sulphonyl)phosphonium toluenesulfonate (a) and its recycled sample after the fourth run (b).

Table 3 Hammett acidity function values of various concentrations of investigated IL

\begin{tabular}{llllll}
\hline Entry & IL (mol\%) & $A_{\max }$ & {$[\mathrm{In}](\%)$} & {$\left[\mathrm{InH}^{+}\right](\%)$} & $H_{0}$ \\
\hline 1 & 0 & 0.361 & 100 & 0 & \\
2 & 5 & 0.347 & 96.20 & 3.80 & -1.40 \\
3 & 6 & 0.345 & 95.59 & 4.41 & -1.46 \\
4 & 7 & 0.329 & 91.22 & 8.78 & -1.78 \\
5 & 10 & 0.315 & 87.28 & 12.72 & -1.96
\end{tabular}




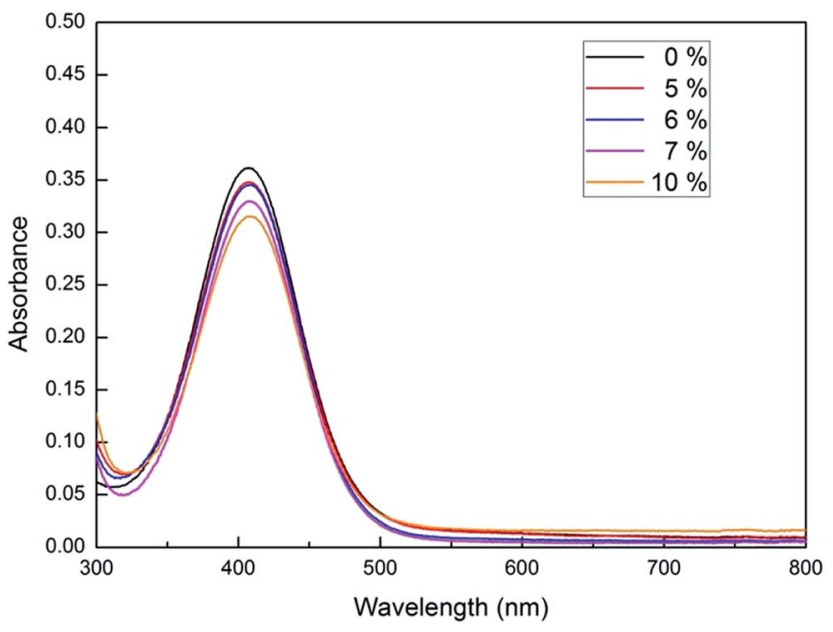

Fig. 3 The UV/Vis spectra of 4-nitrodiphenylamine indicator measured in its cosolutions with IL at different concentrations.

Finally, the presence of phosphonium functional group is confirmed by two concomitant absorption bands at 1121 and $1410 \mathrm{~cm}^{-1}$ corresponding with $\mathrm{P}-\mathrm{Ar}$ and $\mathrm{P}-\mathrm{CH}_{2}$ bond deformation, respectively. A close similarity in pattern between two FT-IR spectra reinforced the above statement on the thermal stability of phosphonium acidic ionic liquid within the temperature range of arylation.

The acidity of triphenyl(butyl-3-sulphonyl)phosphonium toluenesulfonate was examined by means of Hammett acidity function method using 4-nitrodiphenylamine as an indicator. $H_{0}$ values of ionic liquid solutions with a concentration range of $5-10 \mathrm{~mol} \%$ were calculated from the [In]/ $\left[\mathrm{InH}^{+}\right]$ratio which was determined by UV-Vis spectroscopy. Technically, this ratio is directly proportional to the absorbance difference of the indicator in its monocomponent solution and its cosolution with a given quantity of ionic liquid. As can be seen from the Table 3 and Fig. 3 , the $7 \mathrm{~mol} \%$ solution of triphenyl(butyl-3-sulphonyl)phosphonium toluenesulfonate has $H_{0}$ value of -1.78 claiming that this ionic liquid is about 50 times more acidic than [1,2-DiMIMPs][OTs] analogue with an $H_{0}$ value of -0.11 as reported by Yang et al. ${ }^{41}$ It could be noted that two analogues almost resemble

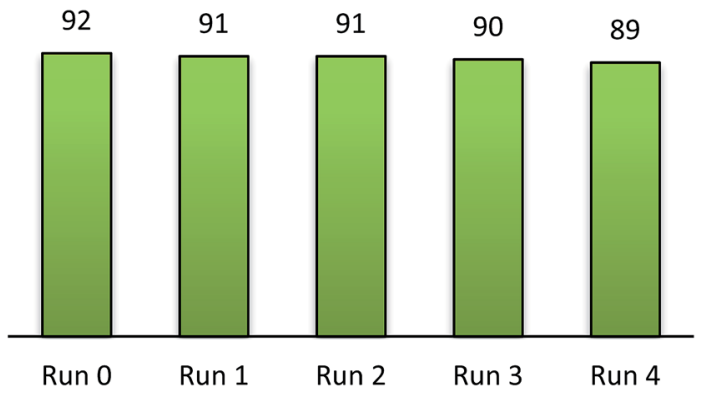

Fig. 4 Recycling test of the catalyst.

each other except their cation centers. For the imidazoliumbased sulfonic acid ionic liquid, the delocalization of positive charge over imidazole ring gives rise to a lessen acidity of the sulfonic acid moiety. Meanwhile, the sulfonic acid functional group tethered to a localized positive-charged center as triphenylphosphonium can behave with more acidic property owing to a better inductive electronwithdrawing effect of positive charge onto Brønsted sulfonic acid group.

The recyclability of triphenyl(butyl-3-sulphonyl) phosphonium toluenesulfonate was surveyed on the optimized arylation of 2 -aminophenol by benzaldehyde. Upon completion of the reaction, the recovered ionic liquid was easily separated from other organic matters by washing many times with diethyl ether. It was subsequently dried in vacuo at $80^{\circ} \mathrm{C}$ for $30 \mathrm{~min}$ before the reuse for consecutive cycles. It can be seen from Fig. 4 that only a very minor loss of catalytic performance was observed over four times of its reuse in the same condensation of benzaldehyde with 2-aminophenol.

A comparative study of the present method with previous literatures was reported in Table 4 . The phosphonium acidic ionic liquid-catalyzed condensation between 2-aminophenol and benzaldehyde afforded the 2-phenylbenzoxazole product in excellent yield under a mild condition without the demand for any additives as in previous reports (Table 4). Furthermore, no loss of catalytic activity in the recycling test of this catalyst is the most prominence of the present method.

Table 4 The comparison of the present method with previous literatures in the synthesis of 2-phenylbenzoxazole

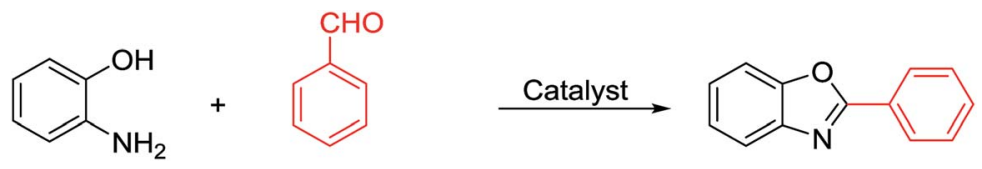

\begin{tabular}{|c|c|c|c|}
\hline Entry & Catalyst & Condition & Yield $(\%)$ \\
\hline 1 & $\mathrm{TiO}_{2}-\mathrm{ZrO}_{2}(10 \mathrm{~mol} \%)$, acetonitrile & $60{ }^{\circ} \mathrm{C}, 15 \mathrm{~min}$ & 91 (ref. 42) \\
\hline 2 & Hf-MOF (1 mol\%), solvent-free & $140{ }^{\circ} \mathrm{C}, 6 \mathrm{~h}$ & 95 (ref. 43 ) \\
\hline 4 & $\mathrm{Sm}(\mathrm{OTf})_{3}(10 \mathrm{~mol} \%)$, ethanol-water & $50-60{ }^{\circ} \mathrm{C}, 2 \mathrm{~h}$ & 92 (ref. 21) \\
\hline 5 & Poly(melamine-formaldehyde) (10 mg), oxygen, toluene & $110{ }^{\circ} \mathrm{C}, 24 \mathrm{~h}$ & 91 (ref. 45) \\
\hline 6 & Present work: triphenyl(butyl-3-sulphonyl)phosphonium toluenesulfonate (7 mol\%), solvent-free & $100{ }^{\circ} \mathrm{C}, 30 \mathrm{~min}$ & 91 \\
\hline
\end{tabular}




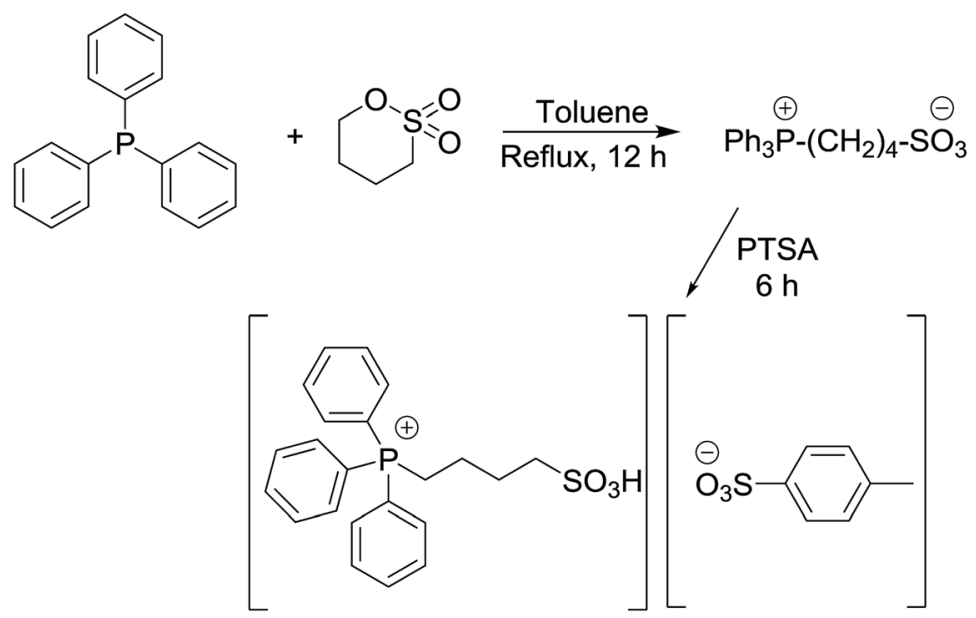

Scheme 1 The synthetic pathway of triphenyl(butyl-3-sulphonyl)phosphonium toluenesulfonate.

\section{Experimental}

\section{Chemicals, supplies, and instruments}

All starting materials were purchased from Sigma-Aldrich and employed without further purification. Silica gel (230-400 mesh) for flash chromatography was obtained from HiMedia Laboratories Pvt. Ltd. (India). TLC (silica gel $60 \quad \mathrm{~F}_{254}$ ) was purchased from Merck. Ethyl acetate (purity $\geq 99.5 \%$ ) and hexanes $(\geq 95 \%)$ were obtained from Xilong Chemical Co., Ltd (China). Chloroform- $d$ (99.8 atom\% D, stab. with Ag) was obtained from Armar (Switzerland). GC-MS spectra were taken on an Agilent GC System 7890 equipped with a mass selective detector Agilent 5973N and a capillary DB-5MS column $(30 \mathrm{~m} \times$ $250 \mu \mathrm{m} \times 0.25 \mu \mathrm{m})$. FT-IR spectra were recorded in the form of $\mathrm{KBr}$ pellets by a Bruker Vertex 70. ${ }^{1} \mathrm{H}$ and ${ }^{13} \mathrm{C}$ NMR spectra were recorded on a Bruker Advance II $500 \mathrm{MHz}$.

\section{Preparation of triphenyl(butyl-3-sulphonyl)phosphonium toluenesulfonate}

Triphenyl(butyl-3-sulphonyl)phosphonium toluenesulfonate catalyst was obtained by the one-pot method as depicted in Scheme $1(97 \%) .{ }^{11-14}$ Its structure was then authenticated by ${ }^{1} \mathrm{H}$ NMR, ${ }^{13}$ C-NMR, FT-IR and TGA. An equimolar mixture of triphenylphosphine and 1,4-butane sultone in toluene solvent (10 $\mathrm{mL}$ ) was stirred and heated at reflux for $12 \mathrm{~h}$ to obtain the zwitterion as a white solid. Then $p$-toluenesulfonic acid was added dropwise to the resulting zwitterion until a separated clear and colorless liquid layer was formed at the bottom of the round bottom flask after approximately $6 \mathrm{~h}$. After completion of the reaction, the crude product was washed with diethyl ether ( 5 $\times 20 \mathrm{~mL}$ ) to remove non-ionic residues. Finally, the ionic liquid was obtained in $97 \%$ yield after the removal of solvent in vacuo.

\section{General procedure for the synthesis of 2-arylbenzoxazole derivatives}

2-Aminophenol (109 mg, $1.0 \mathrm{mmol}$ ) was treated with benzaldehyde (106 mg, $1.0 \mathrm{mmol}$ ) in the presence of triphenyl(butyl-3- sulphonyl)phosphonium toluenesulfonate (20.5 $\mathrm{mg}, 7 \mathrm{~mol} \%$ ) in a $10 \mathrm{~mL}$ glass tube at $100{ }^{\circ} \mathrm{C}$ under solvent-free magnetic stirring. Upon completion of the reaction as indicated by TLC after $30 \mathrm{~min}$, the mixture was diluted and extracted with diethyl ether $(10 \times 5 \mathrm{~mL})$. Then the ethereal solution was washed with water $(2 \times 20 \mathrm{~mL})$ and dried over $\mathrm{Na}_{2} \mathrm{SO}_{4}$. The final product was obtained after solvent removal by a rotary evaporator followed by the purification on a silica gel column chromatography using acetone/petroleum ether (1/19) as an eluent solvent. The structural characterization was performed using ${ }^{1} \mathrm{H},{ }^{13} \mathrm{C}-\mathrm{NMR}$, and GC-MS. The recovered catalyst was reactivated by heating under reduced vacuum at $80^{\circ} \mathrm{C}$ for $30 \mathrm{~min}$ and reused for next cycles.

\section{Conclusions}

In summary, a green and efficient pathway to access to 2-arylbenzoxazoles, 2-arylbenzimidazoles, and 2-arylbenzothiazoles from $o$-aminophenol, $o$-phenylenediamines, and $o$-aminothiophenol, respectively, via the condensation with aldehydes has been successfully developed by outstanding catalytic performance of triphenyl(butyl-3-sulphonyl)phosphonium toluenesulfonate. Not only did this ionic liquid work out on a wide range of substrates and reagents, but it also expressed excellent sustainability of remaining reactivity after four times of recycling. A simple work-up step allowing to isolate the desired products as well as simultaneously recover the ionic liquid catalyst is also an apparent advantage of this process.

\section{Conflicts of interest}

There are no conflicts to declare.

\section{Acknowledgements}

This research is funded by Viet Nam National University, Ho Chi Minh City (VNU-HCM) under grant number 562-2018-18-03. We thank Hoang-Tan Le Doan and Ngoc-Mai Hoang Do for their valuable discussions. 


\section{References}

1 L. A. Nguyen, Q. A. Ngo, P. Retailleau and T. B. Nguyen, Green Chem., 2017, 19, 4289-4293.

2 K. Kuroda, S. Tsuyumine and T. Kodama, Org. Process Res. Dev., 2016, 20, 1053-1058.

3 S. Yamada, K. Murakami and K. Itami, Org. Lett., 2016, 18, 2415-2418.

4 Q. Zhou, J. F. Zhang, H. Cao, R. Zhong and X. F. Hou, J. Org. Chem., 2016, 81, 12169-12180.

5 A. Srivastava, G. Shukla and M. S. Singh, Tetrahedron, 2017, 73, 879-887.

6 D. Wang, J. Albero, H. García and Z. Li, J. Catal., 2017, 349, 156-162.

7 R. Gupta, P. K. Sahu, P. K. Sahu, S. K. Srivastava and D. D. Agarwal, Catal. Commun., 2017, 92, 119-123.

8 M. S. Gerova, S. R. Stateva, E. M. Radonova, R. B. Kalenderska, R. I. Rusew, R. P. Nikolova, C. D. Chanev, B. L. Shivachev, M. D. Apostolova and O. I. Petrov, Eur. J. Med. Chem., 2016, 120, 121-133.

9 R. P. Lester, T. Bham, T. W. Bousfield, W. Lewis and J. E. Camp, J. Org. Chem., 2016, 81, 12472-12477.

10 M. A. Abdelgawad, R. B. Bakr and H. A. Omar, Bioorg. Chem., 2017, 74, 82-90.

11 D. Kim, H. Y. Won, E. S. Hwang, Y. K. Kim and H. P. Choo, Bioorg. Med. Chem., 2017, 25, 3127-3134.

12 W. Zhang, J. Liu, J. M. Macho, X. Jiang, D. Xie, F. Jiang, W. Liu and L. Fu, Eur. J. Med. Chem., 2017, 126, 7-14.

13 K. Chanda, S. Rajasekhar and B. Maiti, Synlett, 2017, 521541.

14 L. Yang and H. Huang, Chem. Rev., 2015, 115, 3468-3517.

15 X.-B. Shen, Y. Zhang, W.-X. Chen, Z.-K. Xiao, T.-T. Hu and L.-X. Shao, Org. Lett., 2014, 16, 1984-1987.

16 F. Zhu, J.-L. Tao and Z.-X. Wang, Org. Lett., 2015, 17, 49264929.

17 L. Tang, X. Guo, Y. Yang, Z. Zha and Z. Wang, Chem. Commun., 2014, 50, 6145-6148.

18 F. Derridj, S. Djebbar, O. Benali-Baitich and H. Doucet, J. Organomet. Chem., 2008, 693, 135-144.

19 J. Roger, S. Mom, M. Beaupérin, S. Royer, P. Meunier, V. V. Ivanov, H. Doucet and J.-C. Hierso, ChemCatChem, 2010, 2, 296-305.

20 J. Azizian, P. Torabi and J. Noei, Tetrahedron Lett., 2016, 57, 185-188.

21 V. Ingle, P. Gorepatil and Y. Mane, Synlett, 2013, 2241-2244.

22 D. Dev, J. Chandra, N. B. Palakurthy, K. Thalluri, T. Kalita and B. Mandal, Asian J. Org. Chem., 2016, 5, 663-675.
23 V. S. Padalkar, B. N. Borse, V. D. Gupta, K. R. Phatangare, V. S. Patil, P. G. Umape and N. Sekar, Arabian J. Chem., 2016, 9, S1125-S1130.

24 R. V. Chikhale, A. M. Pant, S. S. Menghani, P. G. Wadibhasme and P. B. Khedekar, Arabian J. Chem., 2017, 10, 715-725.

25 J. P. Hallett and T. Welton, Chem. Rev., 2011, 111, 3508-3576.

26 A. S. Amarasekara, Chem. Rev., 2016, 116, 6133-6183.

27 K. Goossens, K. Lava, C. W. Bielawski and K. Binnemans, Chem. Rev., 2016, 116, 4643-4807.

28 P. C. Marr and A. C. Marr, Green Chem., 2016, 18, 105-128.

29 H. Niedermeyer, J. P. Hallett, I. J. Villar-Garcia, P. A. Hunt and T. Welton, Chem. Soc. Rev., 2012, 41, 7780-7802.

30 V. I. Parvulescu and C. Hardacre, Chem. Rev., 2007, 107, 2615-2665.

31 M. A. Martins, C. P. Frizzo, D. N. Moreira, N. Zanatta and H. G. Bonacorso, Chem. Rev., 2008, 108, 2015-2050.

32 B. Zhen, Q. Jiao, Y. Zhang, Q. Wu and H. Li, Appl. Catal., A, 2012, 445-446, 239-245.

33 M. A. Martins, C. P. Frizzo, A. Z. Tier, D. N. Moreira, N. Zanatta and H. G. Bonacorso, Chem. Rev., 2014, 114, PR1-PR70.

34 A. Chinnappan, H. Bandal, S. Ramakrishna and H. Kim, Chem. Eng. J., 2018, 335, 215-220.

35 Q. Hou, M. Zhen, L. Liu, Y. Chen, F. Huang, S. Zhang, W. Li and M. Ju, Appl. Catal., B, 2018, 224, 183-193.

36 L. C. Player, B. Chan, P. Turner, A. F. Masters and T. Maschmeyer, Appl. Catal., B, 2018, 223, 228-233.

37 M.-M. Li, C.-S. Duan, Y.-Q. Yu and D.-Z. Xu, Dyes Pigm., 2018, 150, 202-206.

38 K. Kumar, F. Parveen, T. Patra and S. Upadhyayula, New J. Chem., 2018, 42, 228-236.

39 M. Zhang, R. Ettelaie, T. Yan, S. Zhang, F. Cheng, B. P. Binks and H. Yang, J. Am. Chem. Soc., 2017, 139, 17387-17396.

40 A. C. Cole, J. L. Jensen, I. Ntai, K. L. T. Tran, K. J. Weaver, D. C. Forbes and J. H. Davis, J. Am. Chem. Soc., 2002, 124, 5962-5963.

41 J. Yang, H. Zhou, X. Lu and Y. Yuan, Catal. Commun., 2010, 11, 1200-1204.

42 M. R. Patil, J. T. Bhanushali, B. M. Nagaraja and R. S. Keri, $C$. R. Chim., 2018, 21, 399-407.

43 L. H. T. Nguyen, T. T. Nguyen, H. L. Nguyen, T. L. H. Doan and P. H. Tran, Catal. Sci. Technol., 2017, 7, 4346-4350.

44 Y. H. Cho, C.-Y. Lee, D.-C. Ha and C.-H. Cheon, Adv. Synth. Catal., 2012, 354, 2992-2996.

45 D. Yang, P. Liu, N. Zhang, W. Wei, M. Yue, J. You and H. Wang, ChemCatChem, 2014, 6, 3434-3439. 